\title{
"Mixed" Myeloproliferative Neoplasm Due to Co-Occurrence of Different Driver Mutations
}

\author{
Isabelle Plo ${ }^{a-c}$

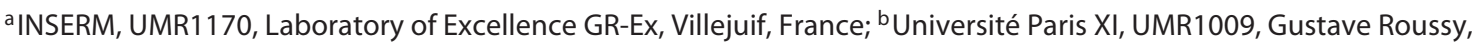 \\ Villejuif, France; ${ }^{\mathrm{C}}$ Gustave Roussy, Villejuif, France
}

\section{Editorial comment on the paper by Elena Tirrò et al. Colony-Forming Cell Assay Detecting the Co-Expression of JAK $2^{\mathrm{V} 617 \mathrm{~F}}$ and $B C R-A B L 1$ in the Same Clone: \\ A Case Report. Acta Haematol 2019;141:261-267}

In the study by Tirrò et al. [1], the authors describe a patient presenting two myeloproliferative neoplasms (MPNs) and harboring a JAK2V617F mutation and a $B C R-A B L 1$ fusion transcript.

MPNs are clonal hematological diseases, which are chronic but can progress to leukemia. They include several entities such as chronic myeloid leukemia (CML), classical MPNs with essential thrombocythemia (ET), polycythemia vera (PV), and primary myelofibrosis (PMF), as well as much rarer entities such as mastocytosis, chronic eosinophilic leukemia, chronic neutrophilic leukemia, and other unclassifiable MPNs. They are all due to genetic abnormalities occurring at the level of the hematopoietic stem cell that induce a constitutive tyrosine kinase activity. CML is the archetype of these diseases and is characterized by an overproduction of granulocytes. This phenotype is due to the translocation $t(9,22)$ corresponding to the Philadelphia chromosome and resulting in the chimeric protein BCR-ABL1. Classical MPNs lead to an overproduction of red blood cells in PV, platelets in ET, and to a deregulation of megakaryocytic and granulocytic lineages in PMF. They are due to mutations in genes that overactivate the JAK2 kinase. Mutations in JAK2 (JAK2V617F) are detected in around 50\%

\section{KARGER}

() 2019 S. Karger AG, Basel

E-Mail karger@karger.com

www.karger.com/aha of the ET and more than 95\% of the PV, and mutations in JAK2 exon 12 are only found in PV. Mutations in the calreticulin gene $(C A L R \mathrm{~m})$ are only found in $25-30 \%$ of the ET and PMF cases, and the mutant CALR constitutively activates JAK2 through binding to the thrombopoietin receptor (MPL). Moreover, 5-10\% of the ET and PMF cases display mutations in the gene encoding $M P L$ (MPL W515K/R/A/K/L) [2].

$B C R-A B L 1$ and non-BCR-ABL1 MPNs are rare with an incidence of $1 / 100,000$ cases, and the frequency of cooccurrence of two disorders in the same patient is even rarer [3]. Tirrò et al. [1] describe a patient presenting with $\mathrm{ET}$ at diagnosis and then 3 years later with CML and harboring both the JAK2V617F mutation and a BCR-ABL1 fusion transcript. Such cases were reported with a $0.2-$ $2.5 \%$ recurrence in large MPN cohorts. Virtually, all combinations of mutations can exist, giving rise to several diseases in the same patient underlying the great heterogeneity of MPNs. The resulting diseases are then driven by each of the different phenotypic mutations. Some of these co-occurrences have been described and include not only $B C R-A B L 1 / J A K 2 \mathrm{~V} 617 \mathrm{~F}$ (CML and PV or ET or PMF); $B C R-A B L 1 / C A L R \mathrm{~m}$ (CML and ET or PMF); $B C R$ $A B L 1 / J A K 2$ exon 12 (CML and erythrocytosis) but also 
Fig. 1. "Mixed" MPNs are extremely rare and correspond to two entities of MPNs that occur in the same patient. For example, CML and ET, or PV, or erythrocytosis, or PMF, or two classical MPNs due to cooccurrence of somatic phenotypic genetic abnormalities (BCR-ABL1, JAK2V617F, CALRm, JAK2 exon 12, and MPLm).

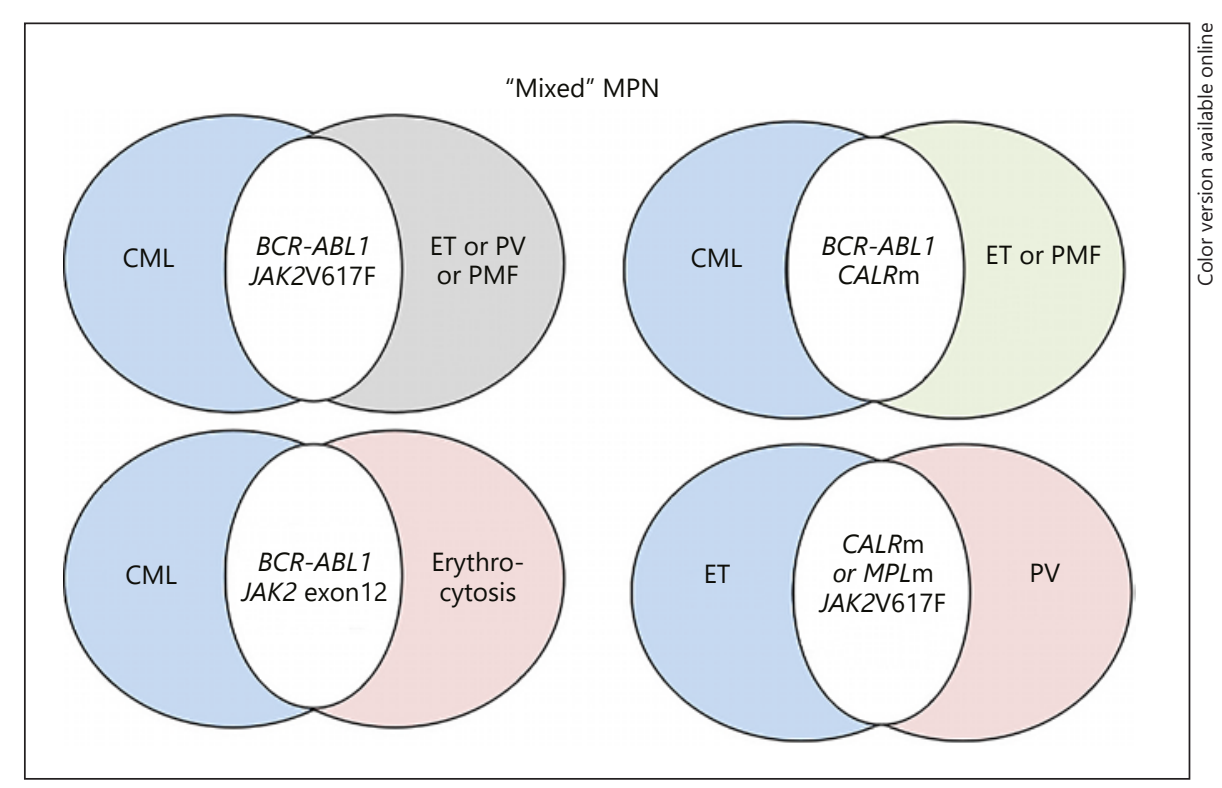

CALRm/JAK2V617F and MPLW515R/CALRm harboring two classical MPNs (Fig. 1).

Interestingly, in the present study, the authors used colony assays to determine the clonal architecture of the genetic abnormalities in the progenitors. This strategy permits one to define if they are in the same clone or not and thus if the diseases are independent or not. The authors describe that $B C R-A B L 1$ arises in a JAK2V617F progenitor, as reported by several other groups that have described CML with an ET, PV, or PMF. Moreover, the same clonal structure was identified with $C A L R \mathrm{~m}$ in a patient with CML and ET [4]. Furthermore, no study has formally established that $B C R-A B L 1$ and $J A K 2 \mathrm{~V} 617 \mathrm{~F}$ mutations could be in two independent clones. In contrast, when CALRm and JAK2V617F were found together (4.2\% of the ET cases), they were in independent clones and remained exclusive. Most of the time and as described in this study, the BCR-ABL1/JAK2V617F clone outcompetes $J A K 2 \mathrm{~V} 617 \mathrm{~F}$ clone alone, showing its high clonal dominance and the dominance of CML over ET. Similarly, when found in the same patient, the CALRm clone outcompetes the JAK2V617F clone, which remains very low ( $<20 \%$ of the cells) [5].

The mechanism of acquisition of several phenotypic drivers in the same patient is still unclear. In fact, the frequency of each individual mutation is rare, so their association would be so unlikely that it is expected that no patient or only extremely few will be found. To explain this paradox, several hypotheses can be postulated. First, a genetic predisposition may exist that can favor either (i)

\footnotetext{
"Mixed" MPN Due to Co-Occurrence of
} Different Driver Mutations the genetic instability and the generation of several mutations in different or similar clones; (ii) the selection of preexisting mutated progenitor cells; or (iii) both. Few predisposing factors have been depicted in classical MPNs. Among them are the 46/1 haplotype, TERT variant and RBBP6 mutations, which deregulate the P53 pathway and induce a potential genetic instability. Moreover, the $A T G 2 B / G S K I P$ locus can predispose to all hematological malignancies, sometimes including $\mathrm{CML}$ and ET in the same patient harboring CALRm and BCR$A B L 1$ [2]. Hematopoietic aging or other acquired associated mutations such as TET2 can also lead to a predisposition to acquire driver mutations. Alternatively, since $B C R-A B L 1$ appears after $J A K 2 \mathrm{~V} 617 \mathrm{~F}$ in this study and others, it could also be hypothesized that JAK2V617Fmediated replicative stress and increased homologous recombination in progenitors could favor genomic translocation such as $B C R-A B L 1$ [6]. Another hypothesis could be that JAK2V617F mutations or BCR-ABL1 translocation could be much more frequent than we think, but that the initiation of a disease by these mutations is a rare event. This hypothesis is supported by the detection of $J A K 2 \mathrm{~V} 617 \mathrm{~F}$ mutations or BCR-ABL1 in the normal population. Therefore, when the disease is already initiated by $J A K 2 \mathrm{~V} 617 \mathrm{~F}$, the probability to get $B C R-A B L 1$ remains the same as in the normal hematopoiesis.

The study by Tirrò et al. [1] also reports the genetic complexity of this case with a branched structure. Indeed, they show evidence indicating the loss of heterozygosity of JAK2 $2617 \mathrm{~F}$ in two independent clones and the detec- 
tion of homozygous JAK2V617F clones with and without $B C R-A B L 1$. This genetic complexity has also been shown in other cases with multiple clones. Moreover, several patients with $B C R-A B L 1$ and $J A K 2 \mathrm{~V} 617 \mathrm{~F}$ can harbor other associated mutations in molecules affecting epigenetics (TET2, ASXL1, and DNMT3A in around 50\%), controlling spliceosome (SF3B1), and in transcriptional factors (P53, RUNX1, PHF6) [3]. Those transcriptional factors, in addition to $J A K 2 \mathrm{~V} 617 \mathrm{~F}$ and $B C R-A B L 1$, could also be involved in the increased genetic instability, resulting in the complex genetic structure of these patients.

In the study discussed here [1], the authors used firstand second-generation tyrosine kinase inhibitors (TKIs; nilotinib, dasatinib) to achieve the complete cytogenetic response for $B C R-A B L 1$, probably without affecting the $J A K 2 \mathrm{~V} 617 \mathrm{~F}$ clone. In this regard, deciphering the order of mutations is of interest, since TKIs will generally target the $B C R-A B L 1$ clone but not JAK2V617F alone, although the second-generation TKIs such as dasatinib were suggested to target both. Novel tailored care and treatment strategies for such patients could be used, such as the combination of TKIs with IFNa, which presents hematological and molecular efficacy both in CML and ET. Alternatively, combining TKIs with ruxolitinib (JAK2 inhibitor) could also be considered in PV or PMF stages for its clinical efficacy.

\section{Acknowledgments}

The author thanks Caroline Marty and William Vainchenker for the critical reading of the manuscript.

\section{Disclosure Statement}

The author has no conflicts of interest to declare.

\section{Funding Sources}

This work was supported by INSERM and grants from INCAPLBio 2015 and 2017, and Labex GR-Ex is funded by the program "Investissements d'avenir."

\section{References}

1 Tirrò E, Stella S, Massimino E, Zammit V, Pennisi MS, Vitale SR, et al. Colony-forming cell assay detecting the co-expression of JAK2V617F and BCR-ABL1 in the same clone: a case report. Acta Haematol. DOI: 10.1159/000496821.

2 Vainchenker W, Kralovics R. Genetic basis and molecular pathophysiology of classical myeloproliferative neoplasms. Blood. 2017 Feb;129(6):667-679.
3 Martin-Cabrera P, Haferlach C, Kern W, Schnittger S, Haferlach T. BCR-ABL1-positive and JAK2 V617F-positive clones in 23 patients with both aberrations reveal biologic and clinical importance. Br J Haematol. 2017 Jan;176(1):135-9.

4 Cabagnols X, Cayuela JM, Vainchenker W. A CALR mutation preceding BCR-ABL1 in an atypical myeloproliferative neoplasm. $\mathrm{N}$ Engl J Med. 2015 Feb;372(7):688-90.
5 Lundberg P, Karow A, Nienhold R, Looser R, Hao-Shen H, Nissen I, et al. Clonal evolution and clinical correlates of somatic mutations in myeloproliferative neoplasms. Blood. 2014 Apr;123(14):2220-8.

6 Plo I, Nakatake M, Malivert L, de Villartay JP, Giraudier S, Villeval JL, et al. JAK2 stimulates homologous recombination and genetic instability: potential implication in the heterogeneity of myeloproliferative disorders. Blood. 2008 Aug;112(4):1402-12. 